\title{
Properties of Pd nanograins in C-Pd composite films obtained by PVD method
}

\author{
M. KozŁowski ${ }^{1 *}$, E. Czerwosz $^{1}$, J. RAdomska $^{1}$, K. SobCZAK ${ }^{2}$, P.A. DŁuŻEwski ${ }^{2}$ \\ ${ }^{1}$ Tele \& Radio Research Institute, Ratuszowa 11, 03-450 Warsaw \\ ${ }^{2}$ Institute of Physics PAS, al. Lotników 32/46, 02-668 Warsaw
}

\begin{abstract}
Properties of palladium nanograins obtained by sedimentation of a soluted C-Pd film prepared by PVD method are presented. These properties were studied using SEM and TEM methods. Dissolved films were prepared by PVD method and after dissolving, they were fractionated to obtain different parts classified with palladium nanograins diameters. Several classes of diameters were determined: below $20 \mathrm{~nm}$, between 20 and $100 \mathrm{~nm}$ and above $100 \mathrm{~nm}$. The defects and triple junction were observed. Multishell carbonaceous structures were found in the big and medium size Pd nanograins.
\end{abstract}

Keywords: Pd nanograins; PVD; defects

(C) Wroclaw University of Technology.

\section{Introduction}

Metal nanoparticles are a key to activity of many catalysts that are important for many practical purposes (catalytic converters, decomposition of molecules). Especially interesting, because of catalytic properties, are the Pd nanograins. Their properties depend on such features as shape, size, defects and facets on the surface. It was found that a great improvement of chemical activity could be achieved depending on the facets on the surface [1]. Many laboratories are looking for better forms of palladium nanograins that could be applied as catalysts for various chemical reactions, such as hydrogen storage compounds as well as components of some powders with tailored mechanical features.

Nanocrystalline metals with a grain diameter less than 30 nanometers exhibit a range of new and interesting deformation mechanisms. Zhiwei et al. [2] reported that for nanocrystalline metals, dislocation sources and pile-ups are not expected to exist within the individual grains because of the limited grain size and the much higher fraction of grain boundary (GB) atoms. Yip [3] proposed that GB-mediated plasticity substitutes for

\footnotetext{
*E-mail: miroslaw.kozlowski@itr.org.pl
}

conventional dislocation nucleation and motion as a dominant deformation mechanism when grain sizes are reduced below a certain value. Additionally, it was found that atomic configuration at the grain boundaries and triple line junctions have an influence on amorphization reaction of a solid [4]. Divakar et al. [5], studying a thin film composed of palladium nanograins, did not find a "gas-like" disorder at these palladium nanograins and they found twinned nanocrystals with fcc structure. The interplanar spacing for (111) planes, determined by HRTEM studies, was $0.24 \pm 0.01 \mathrm{~nm}$. Such effects are also important because they affect many properties of materials (e.g. mechanical properties, such as plasticity and hardness, hydrogen absorption ability, reactivity toward many compounds [6]).

The results of HRTEM studies of nanograins of palladium obtained from a C-Pd film prepared by Physical Vapor Deposition (PVD) method followed by thermal treatment of the sediment of this film solution in toluene/ethanol mixture are presented. Pd nanograins of nanometer sizes were found in HRTEM images of samples prepared by sedimentation. The observed defects were related to the size of Pd nanocrystals. For many Pd nanograins, surrounding carbon shells were found. In the previous paper [7] we observed increased 

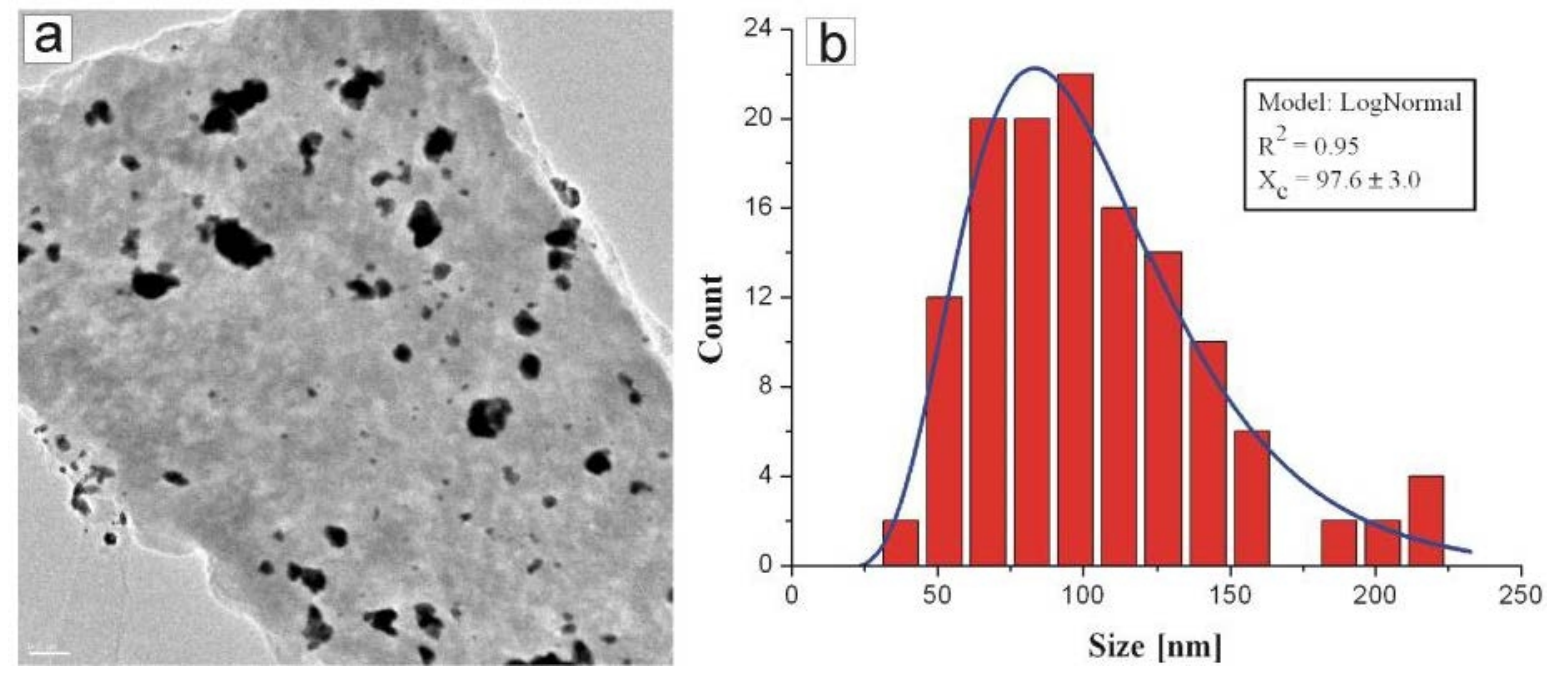

Fig. 1. Big sized Pd nanograins (a) TEM image, (b) histogram of the size of Pd nanograins.
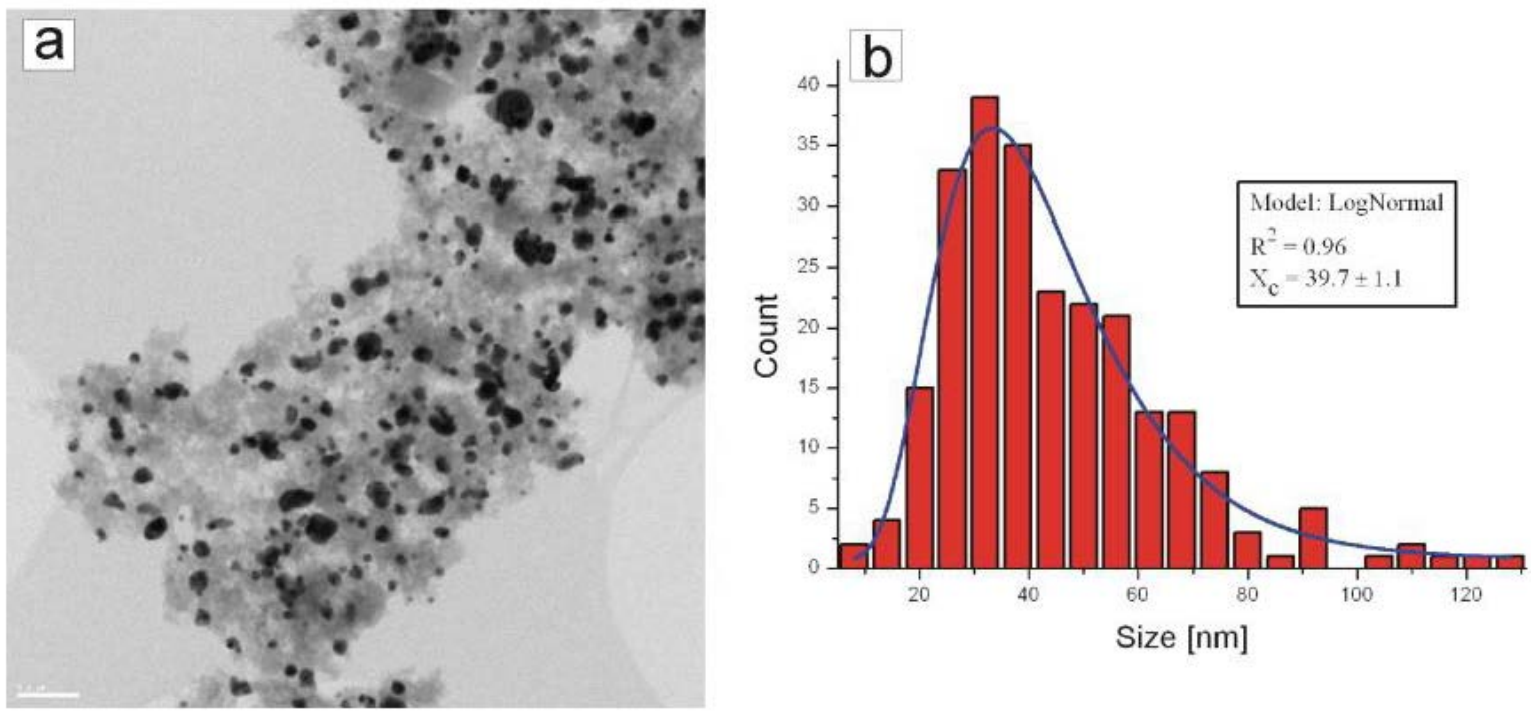

Fig. 2. Medium sized Pd nanograins (a) TEM image, (b) histogram of the size of Pd nanograins.

temperature migration and agglomeration of very small $\mathrm{Pd}$ nanograins (few $\mathrm{nm}$ in diameter), while these nanograins were pure palladium grains without any external shells. When Pd grains are surrounded by carbonaceous shell such effects do not occur. In our study [7] it was also shown, that even for such small nanograins as those ones, having diameter below $20 \mathrm{~nm}$, the formation of defects and twinnings were observed. Palladium nanograins obtained by PVD method are suitable for application as a hydrogen storing component and hydrogen sensing elements. They could be deposited on different surfaces, improving their superficial properties. Therefore, it is important to know a structure of prepared palladium nanograins.

\section{Experimental}

Pd nanograins were obtained by the originally elaborated method. The process was based mainly on PVD method in which a carbonaceouspalladium film (C-Pd film) was obtained by evaporation from two separated sources containing pure $99.95 \%$ fullerene $\mathrm{C}_{60}$ (first source) and 

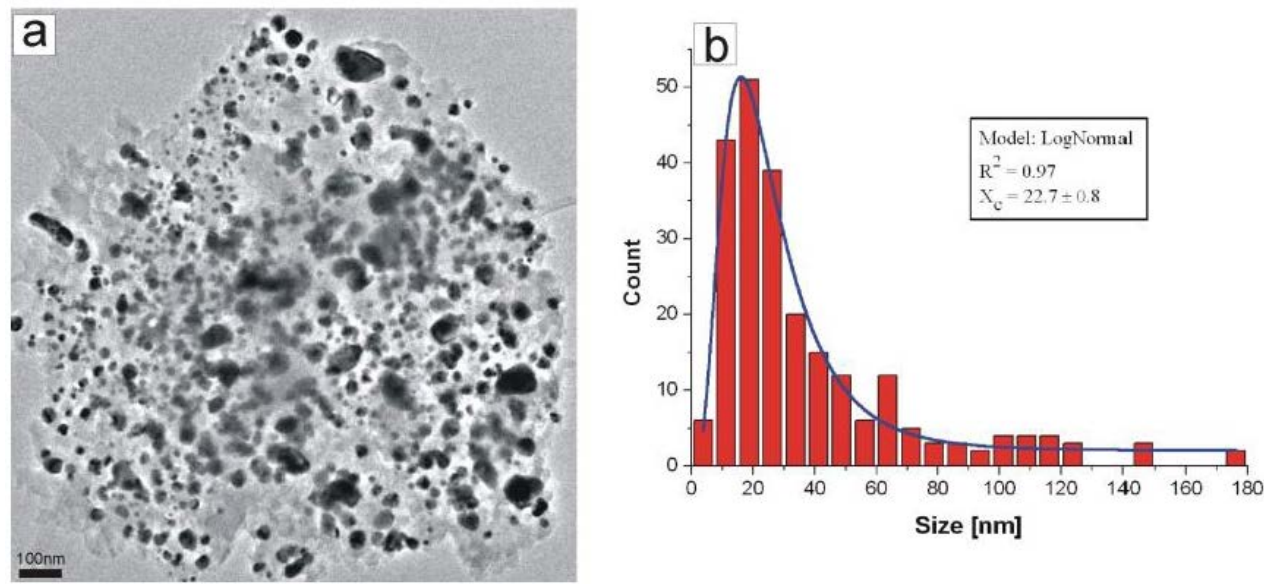

Fig. 3. Small sized Pd nanograins (a) TEM image, (b) histogram of the size of Pd nanograins.
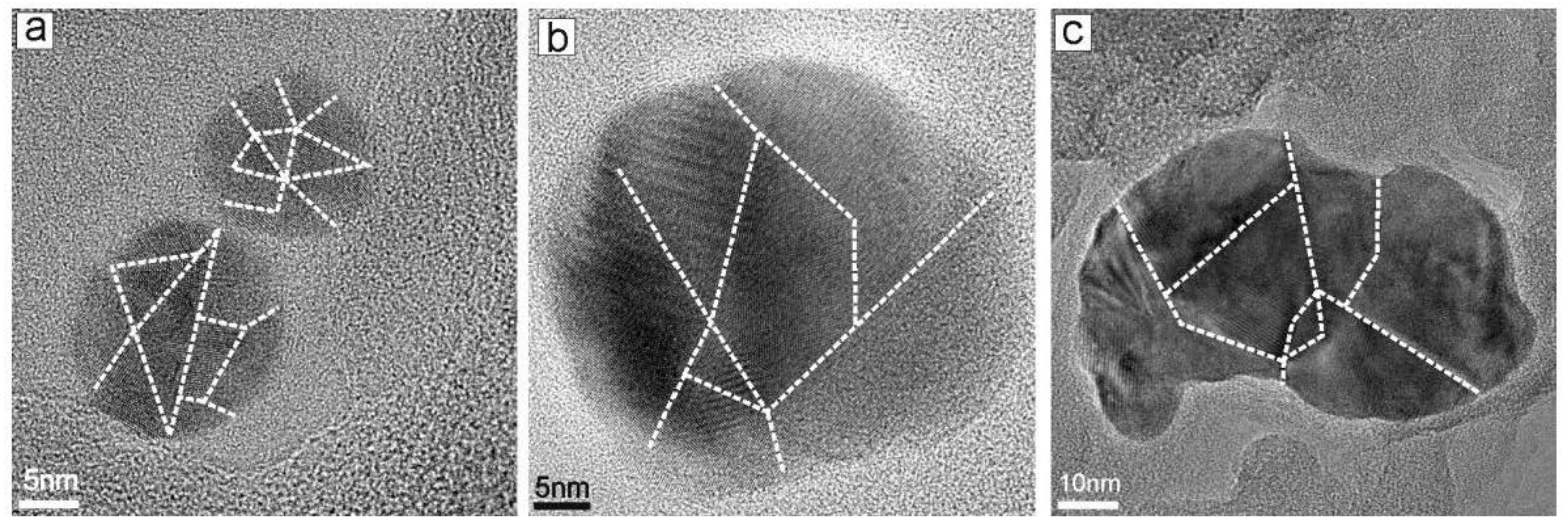

Fig. 4. HRTEM images of Pd nanograins for (a) small class, (b) medium class, (c) big class. Schematic sketch of possible multigrain substructures are over-layered (white dashed lines).

palladium acetate (second source), in dynamic vacuum $\left(10^{-4} \mathrm{~Pa}\right)$. The film was removed from the substrate and dissolved in toluene/ethanol mixture (1:1). Different fractions of Pd nanoparticles, depending on nanoparticle size, were separated by sedimentation process. Then, the suspension was dried, deposited on a silicon plate and annealed at $650{ }^{\circ} \mathrm{C}$ in an atmosphere of argon for 30 minutes to remove carbon deposits. Samples prepared in such a way, containing mostly Pd nanoparticles (and trace of carbon deposit) were investigated by HRTEM.

HRTEM was applied to study details of the structure of Pd nanoparticles. These investigations were performed with the Titan 80-300 Cubed high resolution transmission electron microscope operating at $300 \mathrm{keV}$ incident electron beam energy.

\section{Results and discussion}

The chemical procedure of sedimentation of a film soluted in toluene/ethanol mixture with different fraction of Pd nanoparticles led to obtaining samples containing $\mathrm{Pd}$ nanoparticles of different sizes. These samples were divided into three classes: (1) with small diameter of Pd nanograin: up to $20 \mathrm{~nm}$ nanoparticles; (2) with medium diameter in a range of 20 to $100 \mathrm{~nm}$ and (3) with big diameter: more than $100 \mathrm{~nm}$. This classification resulted from HRTEM studies of the size distribution of Pd nanoparticles in sediment samples. The 


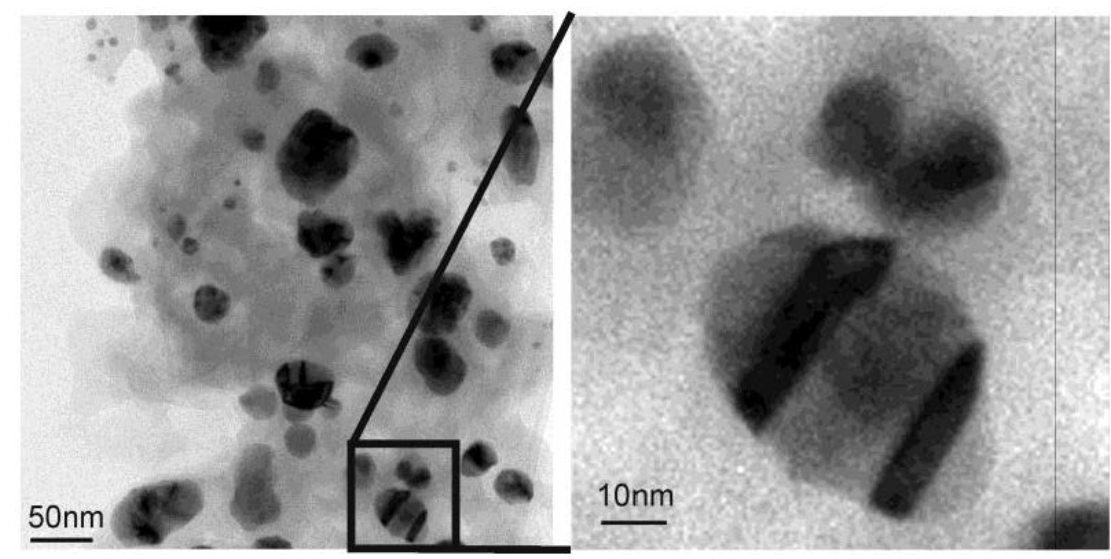

Fig. 5. Pd nanograins with twins.
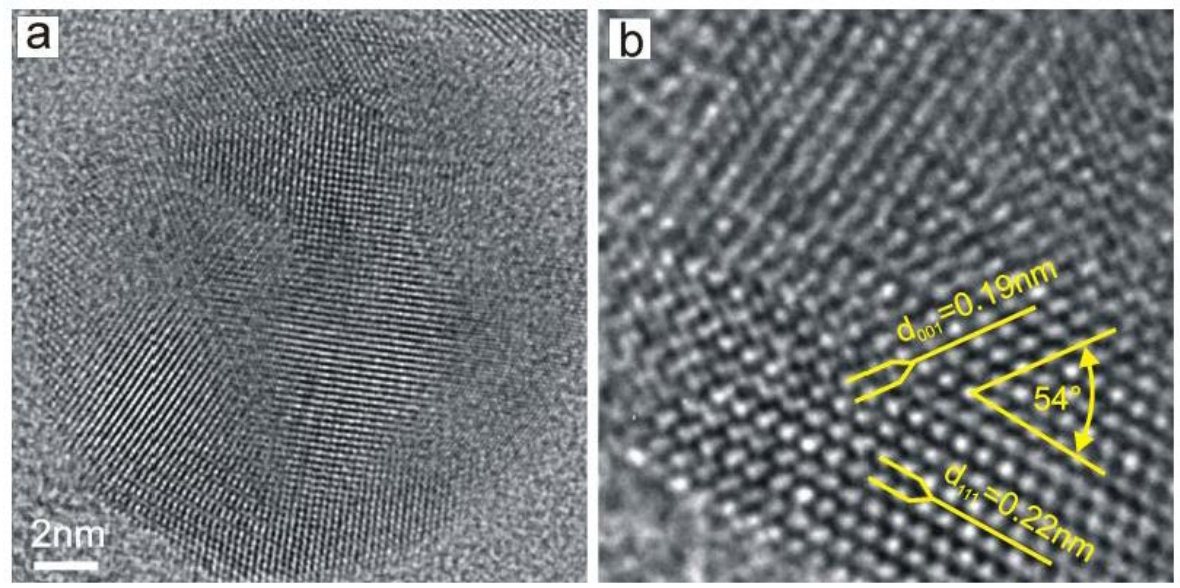

Fig. 6. HRTEM image showing (a) regions of Pd nanograins with different crystallographic orientations (b) area with the interplanar spacing of $0.22 \mathrm{~nm}$ and $0.19 \mathrm{~nm}$.

histograms of size distribution for the samples were obtained and they are presented in Fig. 1 - 3 (in b insets).

In Fig. 1a, 2a and 3a TEM images of the sediment samples are presented. The analysis of the size distribution leads to a conclusion that the $\mathrm{Pd}$ nanograins can be assigned to the above mentioned classes (defined by the average size).

In Fig. $4 \mathrm{Pd}$ nanograins representing these three classes are shown. These HRTEM images show the polycrystalline character of the Pd nanograins. In these images, a schematic sketch of possible multigrain substructures of large features in bright image are over-layered.
In the class of small sized nanograins an average particle size is $20 \mathrm{~nm}$. The smallest nanoparticles are characterized by distinct boundaries between the areas of different crystallographic orientations. Fig. 4a presents two $\mathrm{Pd}$ nanograins from the "small" class. The regions of different crystallographic orientations are clearly visible (over-layered sketch). This image is a superposition of inverse Fourier-filtered image and TEM image. Such superposition improves the quality of interfaces between the regions with different orientations.

In the class of nanograins described as "medium", the boundaries between fragments of different crystallographic orientations (Fig. 4b) are 
visible. The average size of the grains is about $40 \mathrm{~nm}$.

In the class-size of nanograins labeled as "big" an average particle size is $\sim 100 \mathrm{~nm}$. An example of a nanograin representing this class is shown in Fig. 4c. The thickness of the nanograins is too large to accurately characterize the intergranular boundaries. More lines and multigrain substructure of this large $\mathrm{Pd}$ nanograin are visible (Fig. 1a). Between these big and medium classes of Pd nanograins we found twinned nanograins (Fig. 5).

In Fig. 6a a lattice image of the smallest Pd nanograin (diameter $\sim 10 \mathrm{~nm}$ ) is presented. The (111) lattice planes that correspond to interplanar spacing $\mathrm{d}_{111}=0.2244 \mathrm{~nm}$ and (001) lattice planes that correspond to interplanar spacing $\mathrm{d}_{001}=0.1945 \mathrm{~nm}$ are shown. A few triple junctions between regions with different orientations are also observed in this HRTEM image (Fig. 6a). Such junctions were visible for all classes of grains. Such triple junction structures can make an important contribution to the mechanical and physical properties of polycrystalline materials [8-10]. It was shown that in nanocrystalline materials, with grain size less than $10 \mathrm{~nm}$, grain boundary phenomena strongly depend on the triple junction form. Wang et al. [11] showed the results of HRTEM studies of the triple junction in nanocrystalline palladium. They discussed their results regarding grain boundary distortions in connection with the stress accommodation at the junction. They found that the stress concentration at the junction can be released through the twin boundary distortion. In our sample of small Pd nanograins we observed junctions. We suggest that in our small Pd nanograins the generated stress is released by deformation of the lattice rather than by twins.

In Fig. 6b TEM image showing the area with the interplanar spacings of $0.22 \mathrm{~nm}$ and $0.19 \mathrm{~nm}$ is shown.

In Fig. 7a carbon shells surrounding Pd nanograins are presented. Such shells have not been observed for all Pd nanograins. We observed such effect for many samples of C-Pd films [12, 13], but its origin is still not clear. We suppose that formation of such carbon shells (their number, defects and thickness) depends on such technological parameters as local temperature and palladium concentration in the film. These parameters depend on the geometry of PVD process (source-substrate distance) and temperature of the sources containing fullerenes and palladium acetate. These shells were observed for the medium and big nanograins. Now, there is no evident explanation why we have not observed such shells for small palladium nanograins. We suppose that time is also an important factor. Sedimentation temperature does not change the proportion of numbers of big, medium and small grains.

Trapped lattice dislocations were found in the sample containing medium nanograins. Fig. $7 \mathrm{~b}$ presents such area with a lattice dislocation. This effect could also be connected to the same parameters of PVD process as mentioned above. Higher temperature causes more defects, such as lattice dislocation in surrounding carbon shells. The distance observed between such ordered carbon shells is $\sim 0.33 \mathrm{~nm}$ what corresponds to interplanar spacing between (0002) planes in graphite. When the transformation of fullerenes into such form of carbon, like the observed here shells, is too fast, the dislocations (as this one shown in Fig. 7b) are formed. This happens at higher temperatures.

\section{Conclusions}

We presented the results of HRTEM studies of palladium nanograins obtained by the originally elaborated method. The obtained powder contained three classes of palladium nanograins (big over $100 \mathrm{~nm}$, medium 20 to $100 \mathrm{~nm}$, small below $20 \mathrm{~nm}$ in diameter). We have found that the structure and defects of these nanograins is different for every class. It has also been shown that the grains with big and medium diameters are surrounded by carbon shells, which prevents agglomeration of these palladium nanograins. Small grains do not have such shell and they are highly stressed, what is reflected by many triple junctions observed for these objects. 

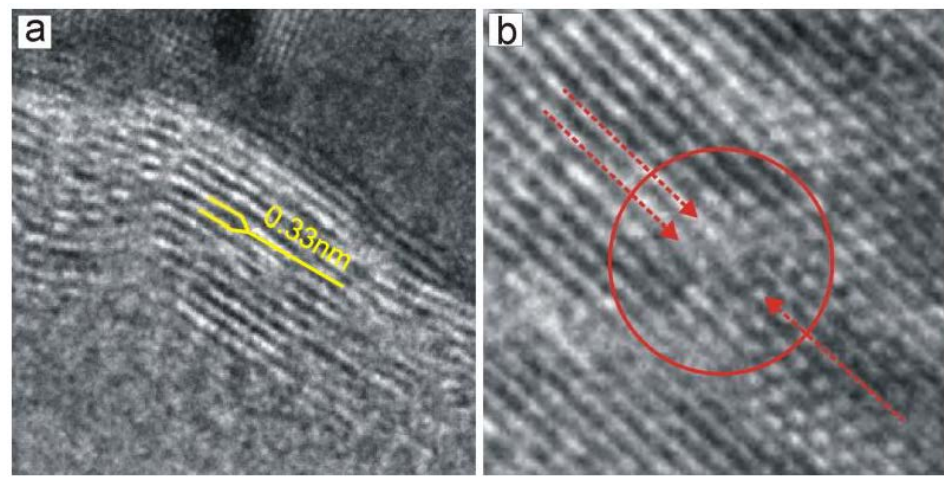

Fig. 7. (a) Carbon shells surrounding Pd nanograin (b) lattice dislocation inside the Pd nanograin.

Sedimentation method allows effective fractionation of the $\mathrm{Pd}$ nanoparticles with different sizes. Each of these fractions may have different physical and chemical properties, taking differences in their structure into account.

\section{Acknowledgements}

This project is co-financed by the European Regional Development Fund within the Innovative Economy Operational Programme 2007 - 2013 (title of the project "Development of technology for a new generation of the hydrogen and hydrogen compounds sensor for applications in above normative conditions" No. UDA-POIG.01.03.01-14-071/08-00).

\section{References}

[1] Habas S.E., LeE H., Radmilovic V., Somorjai G.A., YANG P., Nat. Mater., 6 (2007), 692.

[2] Zhiwei S., Stach E.A., WiezoreK J.M.K., KNAPP J.A., Science, 305 (2004), 654.

[3] YiP S., Nature, 391 (1998), 532.

[4] Sundararaman D., Mater. Sci. Eng. B-Adv., 33 (1995), 307.

[5] Divakar R., Raghunathan V.S., In Fontiers in Material Science, Univ. Press, India, 2005, p. 47.
[6] Bachurin D.V., Gumbsch P., Acta Mater, 58 (2010), 5491.

[7] Sobczak K., DŁużewski P., Klepka M.T., Kurowska B., Czerwosz E., Int. J. Hydrogen Energ., 37 (2012), 18556.

[8] HaCkney S.A., Scripta Metall. Mater., 22 (1988), 1255.

[9] Miura S., Hashimoto S., Fuji T.K., J. Phys. Chem. C, 5 (49) (1988), 599.

[10] Palumbo G., Aust K.T., Scripta Metall. Mater, 22 (1988), 847.

[11] Wang Y., Su Z., PIng D., J. Mater. Sci. Technol., 26 (11) (2010), 1047.

[12] Kowalska E., Czerwosz E, KozŁowski M., Surga W., Radomska J., Wronka H., J. Therm. Anal. Calorim., 101 (2010), 737.

[13] KozŁowski M., Czerwosz E., DŁużewski P., KOWALSKA E., RADOMSKA J., WRONKA H., JAMME, 37 (2) (2009), 304. 